\title{
Anabases
}

ANABASES Traditions et réceptions de l'Antiquité

6 | 2007

Varia

\section{Hittorff, un architecte à l'école de la Grèce}

\section{Adeline Grand-Clément}

\section{(2) OpenEdition}

Journals

Édition électronique

URL : http://journals.openedition.org/anabases/3459

DOI : 10.4000/anabases.3459

ISSN : 2256-9421

\section{Éditeur}

E.R.A.S.M.E.

\section{Édition imprimée}

Date de publication : 1 octobre 2007

Pagination : 135-156

ISSN : 1774-4296

\section{Référence électronique}

Adeline Grand-Clément, "Hittorff, un architecte à l'école de la Grèce », Anabases [En ligne], 6 | 2007, mis en ligne le 01 janvier 2012, consulté le 20 octobre 2019. URL : http://journals.openedition.org/ anabases/3459; DOI : 10.4000/anabases.3459 
Anabases 6 (2007), p. 135-156.

\title{
Hittorff, un architecte à l'école de la Grèce
}

Adeline Grand-Clément

\begin{abstract}
JACQUes-IGNACE HitTorfF (1792-1867) A ÉTÉ Un TÉMOIN mais aussi un acteur privilégié de l'émergence de la science archéologique européenne, au cours de la première moitié du XIXe siècle ; il a pris part à la redécouverte d'une Grèce qui n'est plus abstraite, idéale, mais réelle, diverse et polymorphe ${ }^{1}$. Il incarne le type même de l'artiste-archéologue, ce voyageur curieux, créateur inspiré, érudit polyvalent, qui fréquente autant l'atelier que les champs des ruines antiques et apparaît comme l'une des figures emblématiques de cette période de renouveau. Hittorff est en effet un architecte et urbaniste prolifique et audacieux, qui a laissé sa trace dans le paysage parisien, en même temps que le premier théoricien de la polychromie de l'architecture grecque. Né à Cologne, il se rend à Paris en 1810 et entre à l'École des Beaux-Arts. Il commence sa carrière très tôt, puisqu'en 1818 il est nommé architecte des Menus-Plaisirs du Roi. En 1823-1824, un voyage d'étude en Sicile lui permet d'exercer ses talents d'archéologue sur le terrain et d'observer, sur les vestiges de temples grecs, la présence de couleurs vives. À son retour, il mène une brillante carrière d'architecte, au service de Louis-Philippe, qui le nomme architecte de la Ville de Paris et du Gouvernement, puis de Napoléon III - en dépit de l'hostilité du baron Haussmann, qui n'aime pas cet homme à l'accent germanique. Citons, parmi ses principales réalisations, le théâtre de l'Ambigu comique, le Cirque d'Hiver, la mairie du premier arrondissement, la gare du Nord, ou encore le remaniement de la décoration de la place de la Concorde, des Champs-Élysées et de l'avenue de l'Étoile. Il consacre également une partie de son temps à plusieurs publica-
\end{abstract}

1 L'intérêt porté aux vestiges matériels d'une civilisation donnée, en tant qu'instruments de sa connaissance, remonte au moins au XVIII siècle, le siècle des Antiquaires : cf. È. GRANAymerich, Naissance de l'archéologie moderne, Paris, CNRS Éditions, 1998, p. 23-137. 
tions savantes, dont sa Restitution du temple d'Empédocle à Sélinonte (1851), ouvrage exposant ses théories concernant la polychromie de l'architecture grecque ${ }^{2}$.

Que retient-on aujourd'hui de Hittorff ? Les études qui lui ont été consacrées demeurent peu nombreuses ${ }^{3}$ et le regard porté sur son œuvre, dans les manuels d'architecture contemporains, est souvent mitigé, comme en témoignent les quelques lignes de présentation suivantes, tirées de l'Architecture du XIXe siècle :

On peut considérer Hittorff comme un archéologue médiocre et un architecte sans grande originalité ; du reste, ses diverses tentatives furent peu déterminantes et sa Restitution du temple d'Empédocle à Sélinonte, ou l'Architecture polychrome chez les Grecs (1846-1851), résumé de ses théories, fut vraisemblablement très peu lue. Cependant, il est certain que sa théorie selon laquelle une revalorisation de l'Antiquité était non seulement possible mais infiniment souhaitable, eut une influence considérable sur de très nombreux architectes de diverses tendances [...]. En outre, c'est lui (et presque sans appui) qui rompit l'enchantement du classicisme doctrinaire en France et qui ouvrit la voie à l'exubérance du Second Empire 4 .

En dépit d'un premier jugement fort lapidaire, Robin Middleton et David Watkin créditent finalement Hittorff d'un bel exploit et d'une postérité non négligeable : l'homme serait parvenu à promouvoir l'Antiquité tout en sonnant le glas du néo-classicisme - ce mouvement culturel et artistique né en Europe, à la fin du XVIII siècle, et qui se réclame du modèle antique, en particulier grec. Mais de quelle façon a-t-il pu concilier deux mouvements si contradictoires en apparence? Une telle question invite à analyser les différentes facettes du personnage, en jaugeant la part prise par l'éduca-

2 J.-I. HitToRfF, Restitution du temple d'Empédocle à Sélinonte. L'architecture polychrome chez les Grecs, Paris, Firmin Didot, 1851.

3 En France, la seule publication concernant l'homme et son œuvre reste à ce jour le catalogue de l'exposition qui a eu lieu au Musée Carnavalet en 1986-1987 (Hittorff: un architecte du XIXe siècle, Paris, Musée Carnavalet, 1986). Deux biographies sont également disponibles, l'une allemande, l'autre anglaise : K. HAMmER, Jakob Ignaz Hittorff : ein Pariser Baumeister 1792-1867, Stuttgart, Hirsemann, 1968 ; D.D. SCHNEIDER, The Works and Doctrine of Jacques Ignace Hittorff (1792-1867), New York-Londres, Garland, 1977. La première a été reprise dans un court article par P. LAVÉDAN : "Un Allemand à Paris au XIXe siècle : J.I. Hittorff, archéologue, architecte et urbaniste", Journal des Savants, juil.sept. 1969, p. 173-188. Le choix du titre ne manque pas de surprendre, quand on sait que l'architecte rhénan a composé ses ouvrages en français et a finalement obtenu la nationalité française. Il est inhumé au cimetière Montmartre.

R. Middleton, D. Watkin, Architettura dell'Ottocento, Milan, Electa, 1980, trad. fr. Architecture du XIXe siècle, Paris, Gallimard, 1993, p. 100. L. Hautecoeur présente Hittorff comme " un des premiers architectes qui voulut renouveler le classicisme " (Histoire de l'architecture classique en France, VI, Paris, Picard, 1957, p. 238) et le range dans la catégorie des « architectes sacrés romantiques " qui conservèrent les traditions et les méthodes classiques (p. 228). 
tion classique qu'il a reçue dans le façonnement de sa personnalité d'artiste et de savant, puis en analysant l'apport scientifique de ses recherches et travaux d'archéologue, ainsi que la nature de ses projets et réalisations d'architecture, dans leur rapport au modèle antique.

\section{L'apprentissage à l'ombre des Anciens}

Hittorff, élève à l'École des Beaux-Arts, a bénéficié d'une solide formation classique, comme tous les autres apprentis architectes de la première moitié du XIXe siècle. Lart grec fournissait alors aux artistes un modèle indépassable qu'il s'agissait d'imiter ou dont il fallait s'inspirer, suivant en cela le dogme winckelmannien. Mais son apprentissage a aussi été fertilisé par des lectures, des rencontres et des voyages, qui ont conféré à son parcours un caractère singulier et préparé sans doute pour une part son œuvre scientifique, en l'intégrant à un réseau de savants, aux ramifications européennes.

Hittorff a commencé sa carrière par une première formation à la fois théorique et pratique dans sa ville natale, Cologne. Son père, issu d'une famille d'artisans modestes, l'avait destiné très tôt à la profession d'architecte. Jeune, il a donc étudié le classicisme français avec le collectionneur d'art Ferdinand Franz Wallraf, recteur de l'Université de Cologne, tout en participant à quelques chantiers : dès l'âge de quinze ans, il dessine et construit des maisons. Il décide en 1810 de s'installer à Paris, afin de parfaire ses connaissances et de compléter sa formation. La Rhénanie étant occupée par la France depuis 1794 et Cologne intégrée à un département français depuis 1801, il possède la citoyenneté française et est admis à l'École impériale et spéciale des Beaux-Arts en 1811.

Cette école, fondée par Napoléon en 1806, est née de la fusion de l'École d'Architecture et de celle de Peinture et de Sculpture, héritées de l'Ancien régime. Les futurs architectes y reçoivent un enseignement comprenant des cours de mathématique, de géométrie, de théorie architecturale, d'histoire de l'art et des constructions, ainsi qu'un cours d'histoire générale. La formation dispensée demeure ainsi très théorique et n'inclue aucun cours de pratique professionnelle. Pendant dix ans, les élèves se consacrent à des projets de monuments : on leur demande de produire esquisses, rendus, aquarelles, sans se soucier de leurs talents réels de constructeurs ni de leur aptitude à construire des édifices adaptés aux besoins ${ }^{5}$. Le parcours des élèves est couronné par le prix de Rome : le lauréat du concours obtient une pension lui permettant d'étudier pendant cinq ans à l'Académie de France à Rome. Lors de son retour à Paris, il doit

5 Il faut attendre 1865 pour que soit créée, à l'initiative de l'architecte Trélat et avec l'appui de Viollet-le-Duc, une école centrale d'architecture, entièrement privée, mieux adaptée à la formation des futurs architectes (cf. F. SEITZ, L'École spéciale d'architecture, 1865-1930, Paris, Picard, 1995). 
s'efforcer de participer à des Salons, rechercher des commandes prestigieuses. Il s'agissait de plaire pour réussir, non d'être original, et de se conformer au modèle classique élaboré au sein de l'Académie des Beaux-Arts ${ }^{6}$. Au début du XIXe siècle, cette prestigieuse institution, chargée de donner son avis sur tous les projets ou questions d'art que lui adresse le gouvernement, est dirigée par un grand érudit, Antoine Chrysostome Quatremère de Quincy, secrétaire perpétuel de 1816 à 1849. Ardent défenseur du classicisme et admirateur de l'idéal porté par Johann Joachim Winckelmann, il met un point d'honneur à maintenir dans l'école le prestige de l'Antiquité et insiste sur le rôle de l'archéologie dans la formation des architectes modernes 7 . Les cours de théorie architecturale reposent sur l'étude des formes romaines et, de plus en plus, à la faveur des découvertes récentes, hellènes. On recourt aux relevés et dessins de monuments effectués en Italie ou en Grèce par des architectes voyageurs, ainsi qu’à des reproductions en plâtre d'éléments de façades de temples, à l'instar de ce qui se fait dans le domaine de la sculpture, avec les moulages de statues, dont on admire les courbes parfaites et la blancheur immaculée.

Durant ses années d'apprentissage à l'École, Hittorff subit l'influence du grand Quatremère de Quincy, mais aussi d'autres Académiciens tel Léon Dufourny, spécialiste de l'architecture sicilienne, qui contribuent à éveiller son goût pour l'architecture antique. Il effectue en outre, entre 1820 et 1821, deux voyages d'étude à l'étranger, l'un en Angleterre, l'autre en Allemagne. Le premier lui permet de rencontrer les membres de la Société des Dilettanti, une association d'amateurs éclairés issus de l'aristocratie, fondée à Londres, en 1734, pour l'encouragement des beaux-arts et la promotion de l'Antiquité. Parmi ceux-ci figurent les découvreurs des marbres d'Égine, Charles Robert Cockerell et Charles Barry. À Berlin, lors de son second voyage, il noue des liens avec Karl Friedrich Schinkel, jeune peintre et architecte prussien. Ces contacts témoignent d'une grande ouverture d'esprit et contribuent à son insertion dans des réseaux savants, préparant sa reconnaissance ultérieure au sein de la communauté scientifique européenne.

Au terme de sa formation, Hittorff a acquis une solide connaissance de l'Antiquité. Celle-ci repose d'abord sur une maitrise des sources littéraires: Quatremère de Quincy l'a encouragé à lire assidûment les auteurs anciens. Dans l'ouvrage magistral qu'il publie en 1851, Hittorff annonce que, pour élaborer ses théories sur la polychromie de l'architecture grecque, il a passé en revue les témoignages littéraires des Anciens ; il omet cependant de préciser lesquels ${ }^{8}$. On peut essayer d'en avoir une idée en examinant la composition de son immense bibliothèque, riche de plus de mille quatre cents

6 Les liens demeurent en effet très étroits entre l'Académie et l'École - la plupart des professeurs sont des Académiciens.

7 Cf. R. SCHNEIDER, Quatremère de Quincy et son intervention dans les arts (1788-1830), Paris, Hachette, 1910, p. 206-219.

8 HitTorfF, Restitution du temple d'Empédocle à Sélinonte, p. 5. 
volumes, dont le catalogue a été publié ${ }^{9}$. Le résultat est somme toute décevant : on compte moins d'une dizaine d'éditions ou de traductions d'auteurs latins et grecs. On ne s'étonnera point de voir figurer en bonne place les dix livres de Vitruve, considérés comme la référence sur l'architecture antique. Hittorff en détient une édition latine, ainsi que trois traductions : italienne, française (celle de Claude Perrault) et anglaise. S'y ajoute l'ouvrage de Frontin sur les aqueducs. Hittorff possède également les Images de Philostrate, traduites en français (dans l'édition de 1637, de Blaise de Vigénère) ; la présence de l'ouvrage témoigne de l'intérêt qu'il porte à la peinture antique. D'autres références ne se rapportent pas directement à la formation reçue au sein de l'École des Beaux-Arts : citons les Vies de Diogène Laërce, traduites du grec, et le De Iside et Osiride de Plutarque. La première ouvre lui permet d'identifier un petit temple de Sélinonte ${ }^{10}$; la seconde illustre bien l'intérêt qu'il porte à la civilisation égyptienne ${ }^{11}$. La bibliothèque, enrichie au fur et à mesure de sa longue carrière, a également accueilli une édition latine commentée, parue en 1851-1858, du livre XXXVII de l'Histoire Naturelle de Pline l'Ancien. Dans ce livre, consacré aux gemmes et aux pierres précieuses, le polygraphe romain détaille systématiquement leurs caractéristiques chromatiques : Hittorff y a sans doute puisé de précieux renseignements quant au goût des Anciens en matière de couleurs.

Sa connaissance des realia antiques lui vient davantage des catalogues, guides, récits de voyages et résultats de fouilles qu'il a lus et qui forment une bonne part de sa bibliothèque ${ }^{12}$, ainsi que de la collection d'antiquités qu'il se constitue au cours de son existence ${ }^{13}$. Elle se nourrit aussi de son expérience archéologique personnelle, qui lui permet d'effectuer des observations sur le terrain et de se confronter directement aux vestiges antiques. N'ayant pu se présenter aux épreuves du Grand Prix d'Architecture, le sésame de l'incontournable voyage en Italie, parce qu'il perd la nationalité française

9 G. QUARG, Katalog der Bibliothek Jakob Ignaz Hittorff, Köln, Universitäts und Stadtbibliothek, 1993. La collection a été complétée à la mort de Hittorff par son fils Charles (1825-1898), également architecte. Le catalogue témoigne des multiples pôles d'intérêt du savant, de sa grande érudition et de son excellente maîtrise des langues (allemand, français, italien, anglais, latin ; il connaît moins bien, semble-t-il, le grec ancien). C'est en effet sur la base du témoignage de Diogène (Vies, VIII, 70) qu'il l'attribue à Empédocle. Sur ce petit temple, qu'il découvre lors de son voyage en Sicile, voir plus bas. Son beau-père, Jean-Baptiste Lepère, un architecte parisien, a participé à l'expédition d'Égypte.

12 Cf. G. QUARG, "Archäologische Werke in der Bibliothek von Jakob Ignaz Hittorff", Kölner Jahrbuch für Vor- und Frühgeschichte 25 (1992), p. 49-50.

13 Conservée à Cologne, elle renferme des vases, figurines de terre cuite, fragments de peinture et éléments architecturaux grecs et romains : $H$. von HESBERG, "Die Antikensammlung des Architekten Jacob Ignaz Hittorff”, Kölner Jahrbuch für Vor- und Frühgeschichte 25 (1992), p. 7-48. Une partie de ces objets a été recueillie en Sicile, lors de son exploration archéologique de 1823-1824. 
en $1815^{14}$, il décide, sur les bons conseils de Quatremère de Quincy - qui a lui-même effectué deux voyages, l'un à Paestum, l'autre à Agrigente - de s'y rendre de son propre chef, en sollicitant auprès du roi un congé de son poste d'architecte des Menus-Plaisirs. De 1823 à 1824, en compagnie du dessinateur Ludovic Zanth, il conduit une expédition archéologique en Italie et en Sicile, afin d'y étudier les monuments de l'Antiquité et de la Renaissance. Il s'agit d'un voyage soigneusement programmé et documenté ${ }^{15}$. L'objectif est de vérifier sur le terrain, par lui-même, les informations dont il dispose déjà, et surtout d'y effectuer un maximum de relevés fiables et précis.

Le voyage lui permet d'abord d'élargir le cercle de ses connaissances au sein du milieu savant européen 16. À Rome, il fréquente le grand sculpteur danois Thorwaldsen, chargé de la restauration des sculptures du fronton d'Égine. À Palerme, il a l'occasion de discuter avec Samuel Angell de la décoration peinte des métopes qui viennent tout juste d'être découvertes à Sélinonte, mais ne seront publiées qu'en 1826 : c'est sans doute ce qui décide Hittorff à se rendre au plus vite sur ce site sicilien. Auparavant, il s'arrête un moment à Agrigente. Le lieu abrite en effet le temple monumental de Zeus, alors connu sous le nom de "temple de Jupiter Olympien " et considéré par les néo-classiques comme l'un des symboles de la splendeur de l'architecture grecque. L'édifice de style dorique, entièrement détruit, avait déjà fait l'objet de plusieurs études, en particulier de la part de l'architecte britannique Charles Robert Cockerell et d'un jeune rival allemand de Hittorff, Leo von Klenze, qu'il avait eu l'occasion de rencontrer lors de son passage à Rome. L'obtention d'un permis de fouilles lui permet d'effectuer des relevés minutieux et de proposer une nouvelle reconstitution du temple, qui reçoit un accueil assez favorable chez les hellénistes. Mais les plus grandes découvertes ont lieu à Sélinonte, où il met au jour, sur l'Acropole, à côté des trois grands temples connus, un petit édifice ruiné qu'il interprète comme un heroôn dédié à Empédocle - celui que les archéologues modernes connaissent aujourd'hui sous le nom de « temple B 17 ». Il observe et relève de nombreux vestiges de couleurs sur les fragments architecturaux qu'il étudie 18 .

Lorsqu'il rentre de Sicile, il est devenu un helléniste convaincu. À l'instar de Winckelmann et de Quatremère de Quincy, il exalte désormais la primauté de l'art des

14 En 1815 a lieu le Congrès de Vienne qui rattache la Rhénanie à la Prusse. Hittorff sera finalement naturalisé français en 1842 . H. G. NiEMEYER, "Hittorff, historien d'art et archéologue", in Hittorff, p. 319.

Sur le détail du voyage en Italie et en Sicile, des étapes et des rencontres qui jalonnent le trajet, voir M. COMETA, "L'architettura italiana tra policromia e stroricismo", in M. Tatti, Italia e Italie. Immagini tra rivoluzione e restaurazione, Roma, Bulzoni, 1999, p. 299-325. Ce temple, daté du IVe ou du III siècle avant notre ère, était peut-être consacré à Asclépios. Sur l'activité archéologique de Hittorff en Sicile, cf. NIEMEYER, "Hittorff, historien d'art et archéologue", in Hittorff, p. 319-321. 
Grecs sur celui des Romains ${ }^{19}$. Pour autant, la profonde admiration qu'il éprouve pour la beauté et la perfection de l'art grec ne l'aveugle pas, et ses propres découvertes concernant la polychromie de l'architecture grecque vont d'une certaine façon contribuer à ébranler les fondements de l'idéal classique, reposant jusque-là sur une vision blanche et épurée de l'hellénisme.

\section{Une nouvelle image de l'architecture grecque : la révélation et l'acceptation de la bigarrure antique}

Hittorff peut être considéré comme l'inventeur de la polychromie de l'architecture antique. S'il n'est pas le premier à envisager la présence de couleurs sur les monuments grecs, il est le premier à rassembler méthodiquement les preuves afin d'en proposer une interprétation cohérente et systématique, qui trouve écho bien au-delà des frontières de la France, non sans susciter débats et controverses 20.

Dans son ouvrage de 1851, Hittorff se présente comme le premier à avoir élaboré une théorie générale sur la polychromie de l'architecture grecque : il affirme qu'aucun ouvrage d'archéologie ou d'histoire de l'art n'a jusque-là traité de la question ${ }^{21}$. Selon lui, en dehors des rares auteurs à avoir signalé la présence de peinture sur les édifices et leur parure sculptée 22 - à savoir Johann Martin Wagner et Friedrich Wilhelm Joseph

19 Soulignons que ni Winckelmann, ni Quatremère de Quincy, ni Hittorff n’ont effectué de voyage en Grèce.

20 Pour une histoire générale de la découverte de la polychromie de l'art grec, cf. M.-F. BILlOT, "Recherches aux XVIIe et XIXe s. sur la polychromie de l'architecture grecque", in Paris-Rome-Athènes, le Voyage en Grèce des architectes français aux XIXe et XXe s., Paris, École Nationale des Beaux-Arts, 1982, p. 61-125 et A. PrATER, "The Rediscovery of Colour in Greek Architecture and Sculpture", in M. A Tiverios et D.S. TsiafaKis, Color in Ancient Greece, Thessalonique, Aristotle University of Thessaloniki, 2002, p. 23-36. Nous avons également consacré un article à cette question, en analysant l'origine des résistances qui obèrent l'acceptation de la couleur dans la tradition néo-classique : "Couleur et esthétique classique au XIXe siècle : l'art grec antique pouvait-il être polychrome ?”, Íthaca. Quaderns Catalans de Cultura Clàssica 21 (2005), p. 139-160. HitTORfF, Restitution du temple d'Empédocle à Sélinonte, p. 5-6 (il ajoute : "Ce fait n'arrêta pas même Winckelmann ; il resta inconnu comme avant ce grand archéologue. ")

Dans cet inventaire un peu succinct, Hittorff omet de signaler des publications plus anciennes qui font elles aussi état de vestiges de couleurs sur les édifices grecs : les Antiquities of Athens de deux Anglais, le peintre J. Stuart et l'architecte N. Revett, qui arpentent la Grèce de 1751 à 1753 afin de rassembler des modèles de dessins architecturaux pour la société des Dilettanti, les observations de J.-B. Fauvel et du diplomate français Choiseul-Gouffier, à la fin du XIXe siècle, dont le Voyage pittoresque de la Grèce figure dans la bibliothèque de Hittorff, ou encore l'article de Cockerell sur Égine, qui paraît à l'occasion de la restauration des marbres entreprise par Thorwaldsen ("On the Aegina Marbles", Journal of Science and the Arts 6 (1819), p. 340-341). 
Schelling 23, Otto Magnus von Stackleberg 24 et Eduard Dodwell 25 - le seul à avoir abordé la question de la polychromie est Quatremère de Quincy, dans sa publication magistrale de 1814, Le Jupiter Olympien 26. L'œuvre est effectivement pionnière, puisqu'elle affirme avec force l'engouement qu'éprouvaient les Grecs pour les sculptures polychromes, auxquelles l'assemblage de matières précieuses et nobles telles que l'or et l'ivoire procurait un chatoiement splendide et admirable. La technique chryséléphantine, maniée avec art par Phidias, en fournit une preuve indiscutable : Quatremère de Quincy propose ainsi dans les planches de son ouvrage quelques reconstitutions de statues monumentales polychromes, telle celle de Zeus à Olympie. Centrée sur la sculpture, l'étude n'évoque que brièvement l'existence d'une polychromie architecturale antique : l'auteur se borne à mentionner les traces observées par les voyageurs sur le Parthénon et le temple d'Héphaïstos (le "Théseion ») sur l'agora d'Athènes, annonçant son intention de se pencher sur le sujet ultérieurement ${ }^{27}$ - dessein qu'il n'a finalement pas mis à exécution.

Hittorff reprend ainsi le flambeau, en transférant la question de la polychromie du domaine de la sculpture vers celui de l'architecture, du domaine des matériaux précieux vers celui de la décoration peinte. Les restitutions aux couleurs fastueuses qui figurent dans l'ouvrage de l'Académicien ont sans doute contribué à éveiller l'imaginaire du jeune architecte, au même titre que les discussions qu'il a eues en Angleterre avec Cockerell et Donaldson, ou à Berlin et à Paris avec Schinkel ${ }^{28}$. Mais c'est surtout son séjour d'étude en Sicile qui joue un rôle déterminant. Les observations effectuées in situ le persuadent de l'existence d'une polychromie généralisée sur les monuments grecs. Le flair du savant se trouve aiguillé dans cette direction par la sensibilité de l'artiste esthète, touché par la beauté des paysages siciliens. Une lettre adressée par Hittorff

23 Tous deux ont eu l'opportunité d'examiner les marbres d'Égine, lorsqu'ils ont été apportés à Rome afin d'y être restaurés.

Ce peintre et archéologue balte, membre de l'expédition scientifique menée en Grèce par Von Hallerstein et Cockerell, de 1810 à 1812, a publié le temple de Bassae en 1826. Dodwell, se fondant sur les découvertes effectuées pour les temples d'Égine et d'Arcadie, admet que la peinture des édifices devait être une pratique largement répandue dans le monde grec, à toutes les époques (A Classical and Topographical Tour through Greece during the Years 1801, 1805 and 1806, London, Rodwell and Martin, 1819, p. 342-343). Le titre se poursuit : L'art de la sculpture antique considéré sous un nouveau point de vue; ouvrage qui comprend un essai sur le goût de la sculpture polychrome, l'analyse explicative de la toreutique, et l'histoire de la statuaire en or et ivoire chez les Grecs et les Romains [...].

A. C. Quatremère De QuincY, Le Jupiter Olympien, Paris, Didot, 1815, p. 30-31. Hittorff cite le passage, ajoutant "Je ne connais aucun ouvrage dans lequel M. Quatremère de Quincy ait donné suite à cette promesse "(Restitution du temple d'Empédocle à Sélinonte, p. 5, n. 1).

M. Cometa estime ainsi que quand Hittorff aborde en Sicile, il est déjà un architecte convaincu de la signification de la polychromie ("L'architettura italiana tra policromia e storicismo", p. 319). 
au peintre Gérard, en 1823 , en témoigne : il y relate de manière vibrante et poétique la vision qu'il a eue un matin, sur le site d'Agrigente. Les premières lueurs de l'aube, nimbant les ruines antiques, font ressusciter devant ses yeux la riche polychromie du temple de Zeus. Il croit apercevoir alors, à l'intérieur de l'édifice au décor opulent, une statue de Junon (Héra) aux couleurs somptueuses, dont la contemplation comble le regard. Un tel spectacle, source de plaisir et enchantement, l'incite à s'affranchir définitivement des " principes puisés dans [ses] lectures et les cours [des] savants antiquaires qui [lui] ont toutes les fois prouvé que le charme des couleurs n'en était plus un en l'adaptant aux arts de la plastique 29 ".

Dès son retour, en juillet 1824, il lit, lors d'une séance publique de l'Académie des Beaux-Arts, un rapport sur son voyage et ses activités scientifiques, afin de présenter ce qu'il considère être sa plus grande découverte, à savoir l'" usage général adopté par les anciens de colorier leurs édifices 30 ». Hittorff entreprend alors de publier le fruit de ses recherches en Sicile, et décide de consacrer un volume aux monuments antiques et un autre aux monuments modernes. Il fait rapidement paraitre, en guise de prélude au premier, un atlas comprenant quarante-neuf planches de gravures 31 , mais il faut attendre l'édition posthume de 1870, achevée grâce au travail de son fils Charles, pour que soit finalement publiée l'Architecture antique de Sicile, ou Recueil des monuments de Ségeste et de Sélinonte ${ }^{32}$.

C'est principalement la grande synthèse de 1851, la Restitution du temple d'Empédocle à Sélinonte, ou L'architecture polychrome chez les Grecs, qui opère une petite révolution au sein du milieu savant. Les théories de Hittorff suscitent une violente polémique, dont le chef de file, en France, n'est autre que Désiré Raoul-Rochette, le successeur de Quatremère de Quincy à la tête de l'Académie depuis 1849. La querelle se prolonge dans les autres pays européens, mêlant philologues, historiens de l'art et archéologues. La vivacité des réactions s'explique par le fait que, si l'ensemble de la communauté des hellénistes admet depuis les années 1830 l'authenticité des traces de couleur découvertes sur les monuments grecs, Hittorff va plus loin, en adoptant une

29 Cité par D. Van Zanten, The Architectural Polychromy of the 1830's, New York-London, Garland, 1977, p. 29.

30 Le texte du discours, conservé sur manuscrit, est reproduit dans Hittorff, p. 336-340. Un extrait du mémoire paraît en 1830, dans les prestigieuses Annales de l'Institut de correspondance archéologique: J.-I. HiTTORFF, "De l'architecture polychrome chez les Grecs, ou restitution complète du temple d'Empédocle dans l'Acropolis de Sélinonte”, Annales de l'Institut de correspondance archéologique 2 (1830), p. 263-284.

31 J.-I. HitTORFF, Architecture antique de la Sicile. Recueil des plus intéressants monuments d'architecture des villes et des lieux les plus remarquables de la Sicile ancienne mesurés et dessinés par J. I. Hittorff, Paris, J. Renouard, 1827.

J.-I. HitTorfF, Architecture antique de la Sicile : Recueil des monuments de Ségeste et de Sélinonte mesurés et dessinés par J.-I. Hittorff \& L. Zanth, suivi de Recherches sur l'origine et le développement de l'architecture religieuse chez les Grecs, Paris, E. Donnaud, 1870. 
approche systématique et totalisante. L'usage des couleurs, loin d'être accidentel ou secondaire, obéissait selon lui à un ensemble de règles composant un système complexe et organique. Ce dernier ne s'appliquait pas uniquement aux édifices siciliens mais aussi à l'ensemble des monuments grecs de l'âge d'or, religieux ou civils. La parure colorée recouvrait l'intégralité de la structure, des scènes historiées occupant les parois murales. Rien n'était laissé dans la couleur originelle de la pierre. Le jeu de la polychromie créait une harmonie générale avec l'environnement naturel et sanctionnait l'alliance parfaite des trois arts (architecture, peinture et sculpture).

Pour donner à voir l'effet général produit par l'application du système polychrome, Hittorff privilégie le recours à des reconstitutions, dans les planches chromolithographiées de ses ouvrages. Il s'agit clairement de convaincre le public de la validité de sa théorie, en essayant de susciter chez le lecteur une forme de plaisir esthétique. Il choisit ainsi, dans l'ouvrage de 1851, de mener à bien l'étude exhaustive d'un édifice, afin d'en proposer une restitution intégrale, du pavement jusqu'au toit. Les données recueillies sur le terrain ne lui suffisant point à inférer l'ensemble de la structure et de l'élévation, ainsi que les modalités d'application des couleurs, il adopte une méthode comparatiste. Ce " comparatisme courageux ", comme se plaît à le qualifier Michele Cometa ${ }^{33}$, frise parfois l'audace sans être toujours rigoureux : c'est précisément l'usage abusif de l'analogie qui prête le flanc aux critiques de ses adversaires, en particulier celles de Raoul-Rochette. Il est vrai que pour élaborer les dessins du temple d'Empédocle, Hittorff a assemblé des éléments hétéroclites, issus d'époques et de lieux différents, générant un amalgame artificiel où se côtoient les peintures étrusques - bien connues depuis la découverte des tombes peintes de Tarquinia, à partir de 1827 - et les trouvailles effectuées à Herculanum et à Pompéi 34 . C'est ce qui gêne le grand helléniste Charles-Ernest Beulé, alors professeur d'archéologie à la Bibliothèque Impériale : s'il reconnait à Hittorff le mérite d'avoir ouvert le débat sur la polychromie de l'architecture grecque, il souligne la nécessité d'envisager le problème dans la diachronie 35 .

Un autre aspect du travail de reconstitution entrepris par le savant rhénan est critiquable sur le plan scientifique : le choix des couleurs relève parfois de l'arbitraire, tant les évidences archéologiques sont, à l'époque, difficiles à interpréter. Pour pallier les

33 COMETA, "L'architettura italiana tra policromia e stroricismo", p. 322.

34 Les fresques pompéiennes sont alors considérées par certains comme des œuvres de piètre qualité, fort éloignées de la virtuosité des grands peintres grecs : c'est l'opinion qu'exprime D. Raoul-Rochette dans la publication qu'il leur consacre : Pompéi, Choix d'édifices inédits. Première partie : Maison du poète tragique à Pompéi publiée avec ses peintures et ses mosaïques, fidèlement reproduites et avec un texte explicatif, Paris, Leconte, 1828, p. 18-19. Hittorff, qui détient un exemplaire de l'ouvrage, l'a annoté, en précisant : "À consulter pour la Restitution du temple d'Empédocle. "

35 Ch.-E. Beulé, "L'architecture au siècle de Pisistrate", Revue générale de l'Architecture 16 (1858), col. 194-199. 
incertitudes, Hittorff s'est laissé porté par ses inclinaisons esthétiques personnelles ${ }^{36}$. Le savant et l'artiste ont travaillé de concert. Une telle collaboration est promise à un bel avenir : les pensionnaires de Rome, enthousiasmés par les propositions de Hittorff, exécutent à leur tour des dessins abondamment colorés, lorsqu'ils tâchent de restituer l'aspect antique des monuments qu'ils étudient, en Italie ou en Grèce. Ainsi, Charles Garnier, en 1852-1853, livre, pour le temple d'Égine, une image richement polychrome, qui fait la part belle à une interprétation très libre des réalités archéologiques 37 .

Appuyé par son ami Antoine-Jean Letronne, qui apporte sa caution scientifique d'helléniste reconnu ${ }^{38}$, et en dépit de l'opposition de Raoul-Rochette, Hittorff obtient finalement d'être élu à l'Académie des Beaux-Arts en 1853, tant pour sa carrière d'architecte que pour ses travaux d'érudition. Il poursuit alors ses recherches dans le domaine de l'archéologie et continue d'exercer sa sagacité afin de faire progresser la connaissance de l'architecture antique et de son histoire. Il se penche notamment sur le témoignage iconographique des peintures murales, susceptible selon lui de pallier les lacunes des sources littéraires et de l'archéologie. L'enquête qu'il entreprend nourrit alors le Mémoire sur Pompéi et Pétra qu'il fait paraitre en 1866 dans le cadre de son travail à l'Institut. Les premières lectures qu'il en donne à l'Académie des Beaux-Arts et à celle des Inscriptions, en janvier 1862, éveillent la curiosité et l'intérêt des membres présents, qui jugent son travail lumineux, au point qu'on lui demande d'en faire paraître une partie dans la Revue archéologique 39 .

Les recherches de Hittorff sur la polychromie rencontrent une audience plus large dans le reste de l'Europe. À Rome, l'archéologue Emil Braun reconnaît dans sa publication de 1851 l'apport le plus significatif à la science archéologique depuis les travaux de Winckelmann ${ }^{40}$. En 1836-1837, lorsque les marbres du Parthénon doivent faire l'objet d'un examen méthodique et scientifique au British Museum, afin de déterminer s'ils étaient peints à l'origine, il est invité pour faire partie de la commission de spécialistes, dans laquelle il retrouve Cockerell ${ }^{41}$. En 1855, il reçoit à Londres la médaille

36 Cf. VAN ZANTEN, The architectural Polychromy of the 1830's, p. 39-40.

37 Sur les envois des jeunes architectes de l'Académie de France à Rome, voir Paris-RomeAthènes, Paris, École Nationale des Beaux-Arts, 1982.

38 Cf. la Lettre d'un antiquaire à un artiste sur l'emploi de la peinture historique murale dans la décoration des temples, que Letronne adresse à Hittorff en 1837.

39 Cf. le témoignage du Bulletin mensuel de l'Académie des Inscriptions du mois de février 1862, publié dans la Revue archéologique, n. s. 5 (1862), p. 214. Hittorff s'exécute dans le numéro suivant: "Pompéi et Petra", Revue archéologique, n. s. 6 (1862), p. 1-18. Il y développe notamment la thèse d'une parenté stylistique entre une peinture de Pompéi et la décoration du tombeau El-Hazane, creusé dans le roc, à Pétra. logica 4 (1852), p. 5.

41 Cf. I. Jenkins, A. Middleton, "Paint on the Parthenon sculptures", Annual of the British School at Athens 83 (1988), p. 183-207. 
d'or du RIBA (Royal Institute of British Architects) pour ses contributions sur l'architecture et l'archéologie classiques. En Allemagne, si Franz Kügler et Leo Von Klenze campent sur des positions plus mesurées ${ }^{42}$, le jeune Gottfried Semper partage les thèses de Hittorff et clame qu'il est le premier à avoir offert une compréhension globale du système polychrome, par ses travaux de reconstitution du temple d'Empédocle, mais aussi, le fait mérite d'être souligné, grâce aux réalisations architecturales qu'il a exécutées à Paris 43.

Il est vrai que la diffusion des travaux de Hittorff ne se limite point au cercle des savants européens. Se sentant investi d'une mission éducative, celle qui incombe selon lui aux artistes 44 , l'archéologue s'emploie à propager le plus largement possible ses découvertes et sollicite alors ses compétences d'architecte. Au salon de l'architecture de Paris de 1830, il expose au public les dessins de ses restitutions polychromes des temples de Sélinonte et d'Agrigente, qui avoisinent les esquisses de projets architecturaux modernes. Lors du salon de 1859, il associe à des relevés et reconstitutions de monuments antiques une maquette de temple grec qu'il a réalisée pour le compte du prince Jérôme, en 1854-1855. Il s'agit d'un cadeau destiné à la tragédienne Rachel, connu sous le nom de "temple des Muses ". L'édifice miniature, d'environ un mètre de haut, richement polychrome, rehaussé d'or et d'argent, abrite la statue de Melpomène, la Muse de la tragédie, identifiée à Rachel. Il possède des métopes vivement colorées et quatre colonnes doriques teintées de jaune en façade. Les murs intérieurs sont ornés de fresques évoquant notamment le sacrifice d'Iphigénie - scène directement empruntée à la Maison du poète tragique à Pompéi. Le peintre Dominique Ingres, ami de Hittorff, a collaboré à la réalisation de la maquette, exécutant la peinture sise à l'arrière, $L a$ Naissance de la dernière Muse 45. L'ensemble, censé reproduire un temple "de l'époque de Périclès ", apparaît clairement comme une réinterprétation en trois dimensions du temple d'Empédocle. La commande princière a donné l'occasion à Hittorff de mettre à profit ce chantier miniature pour poursuivre ses recherches, en endossant le rôle de l'architecte grec.

42 F. Kügler publie en 1835 à Berlin un ouvrage intitulé Über die Polychromie der Griechischen Architektur und Sculptur und ihre Grenzen, dans lequel il défend une vision plus nuancée que Hittorff de l'extension des couleurs sur les édifices grecs. G. Semper, "On the Study of Polychromy, and its Revival", Museum of Classical Antiquities 1 (1851), p. $233 ; 241$. Hittorff considère en effet que l'art n'a pas pour seul but de plaire mais aussi celui d'instruire (SCHNEIDER, The Works and Doctrine of Jacques Ignace Hittorff, p. 214). temple des Muses", in Hittorff, p. 259-264. Une copie a été confectionnée par Isabelle Foriel Destezet à l'occasion de l'exposition "Ingres et l'antique » qui a eu lieu au Musée d'Arles en 2006-2007. 
Ce type d'initiative n'a rien d'exceptionnel dans le contexte européen : il rejoint d'autres pratiques contemporaines similaires, qui ont débuté avec la sculpture 46 et révèlent l'intimité qui existe alors entre le monde des artistes et celui des archéologues. La frontière entre les reconstitutions libres des premiers et les relevés fidèles des seconds n'existe pas encore. Des accointances aussi fortes expliquent la vivacité des résistances aux théories de Hittorff : la révélation de la polychromie antique peut avoir des incidences directes sur les productions artistiques modernes et remettre en cause le règne du courant néo-classique, en obligeant à repenser le rapport au modèle antique 47 .

\section{L'architecture moderne à l'école de l'architecture grecque ? De la bonne façon d'imiter les Anciens}

À la fin du XVIII siècle s'est épanoui en Europe le néo-classicisme, né en réaction contre le mouvement baroque. Il prend pour modèle l'Antiquité, surtout la Grèce ancienne, et joue un rôle moteur dans le développement de l'archéologie, alors conçue comme un moyen d'étendre la connaissance de l'architecture grecque, jusque-là éclipsée par l'architecture romaine ${ }^{48}$. L'Europe se met alors à apprécier les qualités sculpturales du dorique. Jacques Germain Soufflot et son élève Dumont vont relever les temples de Paestum avant d'entreprendre la construction du premier édifice néo-classique de France, à savoir l'église Sainte-Geneviève (futur Panthéon), édifiée entre 1758 et 1789. En Allemagne, ce sont les Propylées d'Athènes qui servent de modèle à la porte de Brandebourg, érigée à Berlin en 1789-1791 par Karl Gotthard Langhonis. En Angleterre, le néo-classicisme, dont l'architecte voyageur Stuart constitue l'un des chefs de file, prend la forme du "Greek Revival », plus précoce, qui met à l'honneur les trois ordres architecturaux grecs, jugés supérieurs aux cinq ordres romains.

Dès lors, les découvertes concernant la polychromie de l'architecture grecque soulèvent la question de savoir si les architectes et sculpteurs modernes doivent imiter les Anciens sur ce point et introduire à leur tour de la couleur dans leurs travaux, rompant ainsi avec le credo néo-classique. Les artistes européens y apportent des réponses diverses. Certains refusent l'intrusion de la couleur, se conformant à l'idéal

46 Citons l'exemple de la grande statue chryséléphantine d'Athéna réalisée par Charles Simiart à la demande du duc de Luynes, et conservée au château de Dampierre. Quatremère de Quincy aurait encouragé l'initiative, sans toutefois pouvoir admirer le résultat, puisqu'il meurt avant son achèvement, en 1855 .

Cf. notre article "Couleur et esthétique classique au XIXe siècle", p. 155-157.

48 Les premiers relevés de temples hellènes paraissent en 1745 dans A Description of the East and some other Countries de R. Pocoke, mais c'est surtout la monumentale publication de Stuart et Revett qui fait date : c'est ce que souligne Hittorff lorsqu'il retrace les débuts de l'archéologie grecque ("Recherches archéologiques en Grèce", Revue archéologique 6 (1862), p. 106). 
esthétique, tout en mesure, défini par Winckelmann, pour qui la plus belle couleur est celle du marbre blanc. D'autres s'enthousiasment pour les nouvelles perspectives offertes par le jeu de la bigarrure et ont à cœur de les mettre à profit ${ }^{49}$. Une voie médiane consiste à tempérer les jeux de couleurs pour les accommoder à la sobriété néo-classique : c'est par exemple celle que choisit d'emprunter Leo von Klenze, lorsqu'il conçoit en 1829 le Walhalla, pour Louis de Bavière ${ }^{50}$.

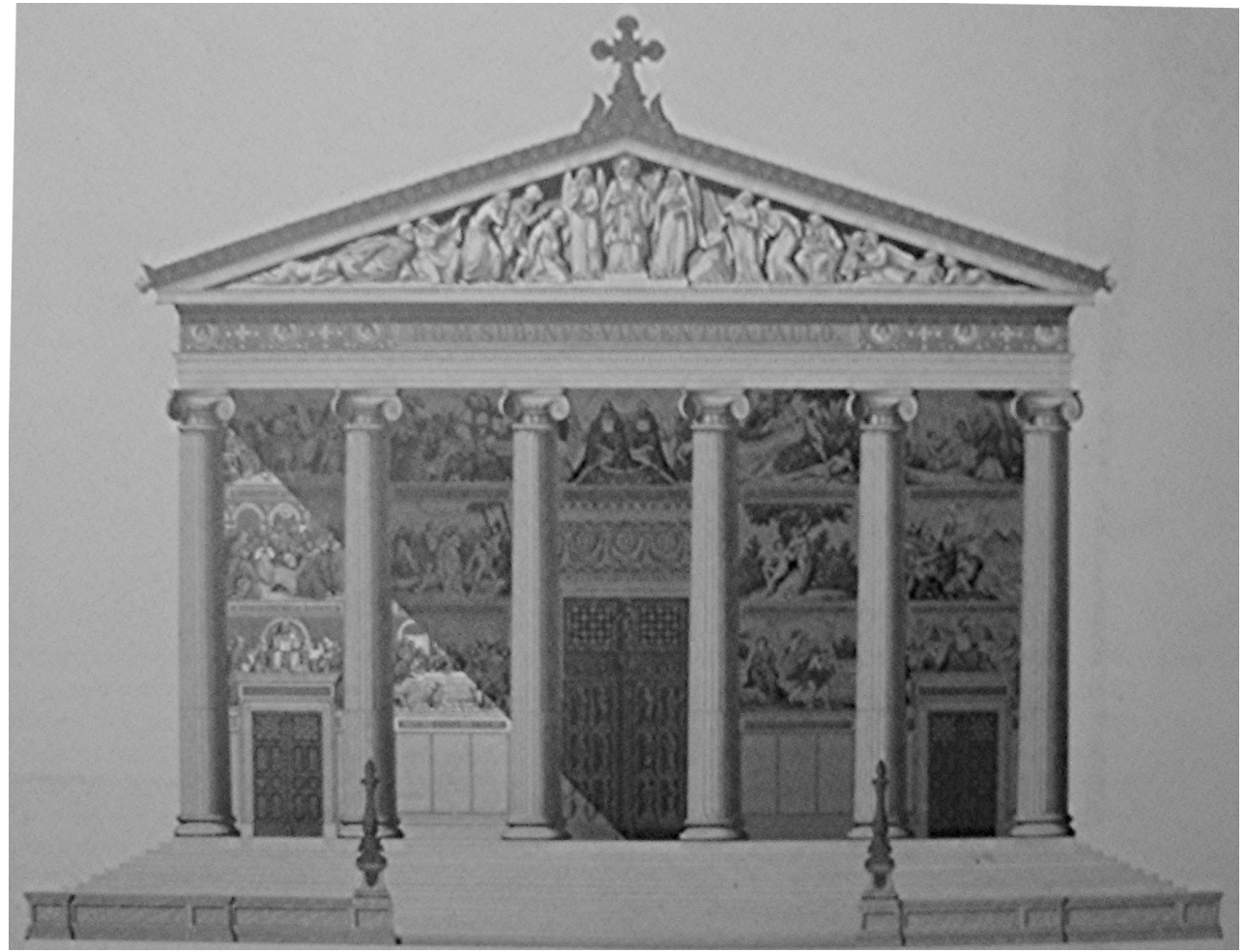

Figure 1. Projet pour la décoration de la façade de l'église Saint-Vincent de Paul, à Paris, au moyen de plaques de lave émaillée (Tiré de J.-I. Hittorff, Restitution du Temple d'Empédocle, 1851, pl. XXIV).

49 Citons les expérimentations d'Owen Jones, en Angleterre, en particulier la coloration des colonnes de la cour du Crystal Palace, qu'il justifie en faisant référence aux découvertes de Hittorff (SCHNEIDER, The Works and Doctrine of Jacques Ignace Hittorff, p. 228).

50 Le monument était destiné à commémorer la défaite de Napoléon à Leipzig en 1813 (Middleton et WatkIn, Architecture du XIXe siècle, p. 100). 
Hittorff fait figure d'inclassable : adoptant une posture qui lui est propre, il utilise toujours la polychromie de façon réfléchie. Sa formation, nous l'avons vu, le destine plutôt à se conformer à la tradition néo-classique. Karl Hammer estime qu'il est allé jusqu'à s'identifier à un architecte grec, "se voyant comme le nouvel Ictinus de l'architecture moderne ${ }^{51}$. " Il est vrai qu'il insère, parmi les nombreuses planches de sa Restitution du temple de Sélinonte, un dessin de la façade polychrome dont il souhaite doter l'église Saint-Vincent de Paul, abolissant les frontières entre passé et présent, entre architecture antique et architecture moderne (fig. 1). Cependant, si Hittorff perçoit effectivement l'Antiquité comme un modèle, source d'admiration, il considère que les architectes doivent également tenir compte des exigences nouvelles de l'ère industrielle et recourir aux techniques et matériaux mis au point grâce aux progrès de la science. C'est ce que révèlent ses réalisations architecturales, qui ne sont jamais des simulacres d'édifices antiques 52 . La polychromie vive qu'il attribue aux monuments grecs ne se retrouve nullement dans l'intégralité de ses travaux ${ }^{53}$; les principes et les procédés en sont réemployés uniquement lorsqu'ils lui paraissent idoines.

Au début de sa carrière, lorsqu'il est architecte des Menus-Plaisirs du Roi, il a pour mission de mettre en scène les fêtes et cérémonies de la cour. Des occasions telles que le sacre de Charles X à Reims, en 1825, se prêtent fort bien à la réalisation de structures temporaires rehaussées de couleurs vives : la richesse de la polychromie devient l'outil de consécration de la magnificence royale ${ }^{54}$. Dans ce cas, toutefois, c'est moins la référence aux usages antiques que la reprise de pratiques médiévales qui peut servir à légitimer ses projets 55 . En revanche, l'influence du modèle grec commence à se percevoir dans le décor chatoyant dont il pare l'intérieur du théâtre de l'Ambigu comique, qu'il réalise avec son collègue Joseph Lecointe en 1828. En 1841, il s'occupe de l'aménagement de la promenade des Champs-Élysées et réalise le premier édifice entièrement polychrome de Paris : le Cirque des Champs-Élysées, rebaptisé plus tard Cirque de l'Impératrice ${ }^{56}$. Hittorff met largement à profit le résultat de ses recherches sur

K. Hammer, "Jacques-Ignace Hittorff 1792-1867", in Hittorff, p. 18.

Il suivait en cela la position adoptée par l'un de ses professeurs à l'École, Charles Percier, architecte en titre de l'empereur, qui soutenait un classicisme plus "libéral " que celui de Quatremère de Quincy (SCHNEIDER, The Works and Doctrine of Jacques Ignace Hittorff, p. 37).

Nulle trace de couleur sur la façade classique de la gare du Nord, dessinée en 1859 et commencée deux ans plus tard, ni sur celle de la mairie du Ier arrondissement, qui fait face à la colonnade du Louvre (1855-1861).

Cf. F. WAQUET, "Architecte des fêtes et cérémonies royales", in Hittorff, p. 27-28.

Rappelons que le XIXe siècle n'est pas uniquement le siècle de la découverte de la polychromie antique, mais aussi celui de la revalorisation de la polychromie gothique (voir à ce sujet les recherches de Viollet-Le-Duc relatives à Notre-Dame de Paris et ses essais de restitution de la polychromie intérieure et extérieure de l'édifice).

Cf. Th. Von JoEst, "Le Cirque d'Été", in Hittorff, p. 171-176. 
Sélinonte : les colonnes corinthiennes sont peintes en jaune et la frise est garnie de rinceaux sur fond bleu, comme sur les métopes découvertes par Angell et Harris 57 . Le bon accueil que les Parisiens réservent à l'édifice $a$, d'après Hittorff, un impact décisif sur l'acceptation par le public de ses théories sur la polychromie grecque. Il continue donc à appliquer le principe d'un décor coloré pour la façade du Cirque de Napoléon, édifié en 1852 et désigné aujourd'hui sous le nom de Cirque d'Hiver. La polychromie d'origine, connue par les descriptions et les dessins de l'époque ${ }^{58}$, avait pour but d'apporter de la gaieté et d'attirer le regard. Elle est aujourd'hui fortement atténuée, en dépit d'une restauration récente (fig. 2). Sur la frise, conçue sur le modèle de celle des Panathénées, les figures sculptées se détachent sur un fond rouge ; Héraklès et des cavaliers y côtoient des jongleurs et autres saltimbanques (fig. 3), illustrant ainsi la curieuse synthèse " gréco-parisienne " réussie par Hittorff.

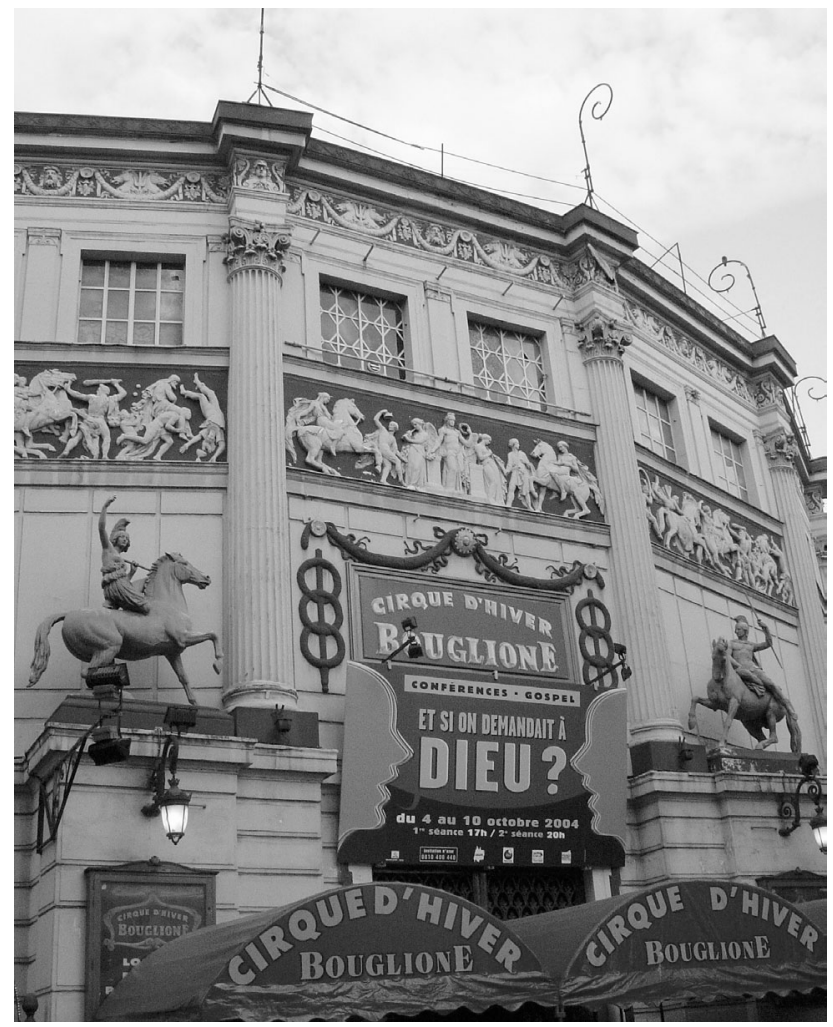

Figure 2. Façade actuelle du Cirque d'Hiver, à Paris (@A. Grand-Clément)

57 Le cirque a été détruit pour l'exposition universelle de 1900 ; pour un dessin en couleurs de la façade de l'édifice, cf. Hittorff, p. 183.

58 Cf. M. Deming, "Le Cirque d'Hiver", in Hittorff, p. 224-225. 


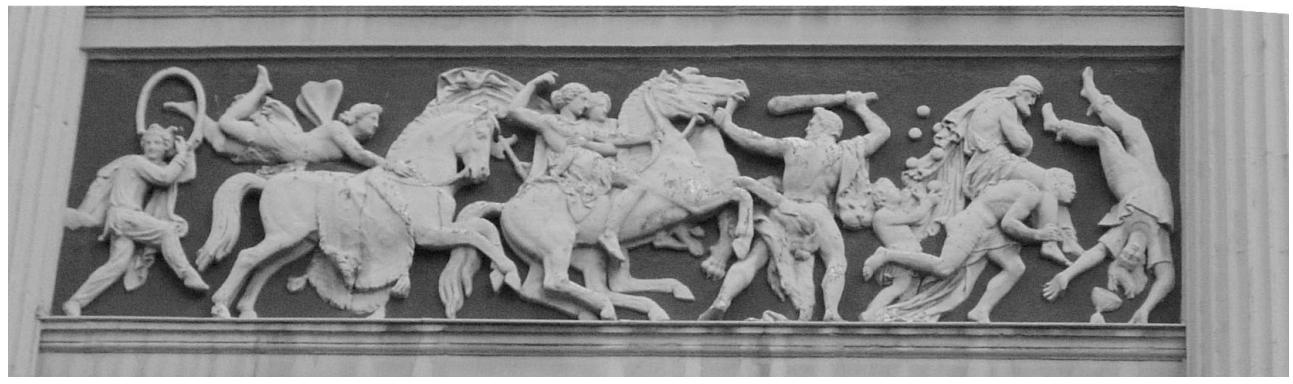

Figure 3. Détail de la frise ornant la façade du Cirque d'Hiver (CA. Grand-Clément)

Si les Parisiens admettent volontiers la présence de la bigarrure sur des édifices de spectacle, ils se montrent plus réservés en ce qui concerne les lieux de culte. Lorsque Hittorff élabore le programme décoratif de l'église Saint-Vincent de Paul, dont la façade rappelle celle d'un temple grec (fig. 4), il doit vaincre des résistances. L'architecte souhaite que la présence des couleurs y revête trois formes différentes : peinture à la cire, peinture sur verre et peinture sur lave émaillée doivent concourir à conférer à l'édifice une splendeur digne de Dieu. Dans la lettre qu'il adresse en 1842 au préfet Rambuteau ${ }^{59}$, afin de se justifier, il explique que son projet a été mûrement réfléchi et résulte de l'enseignement qu'il a retiré de son expérience d'archéologue. Pour autant, Hittorff ne puise pas uniquement dans l'Antiquité pour légitimer sa proposition : les édifices médiévaux, qu'il a relevés en Sicile, méritent tout autant à ses yeux d'être pris en considération. En effet, la liste des "Monuments les plus remarquables des belles époques de l'Art " qui servent de modèle à son projet, écrit-il à Rambuteau, comporte les " immortels ouvrages de la Grèce, de la Rome ancienne " mais aussi les "chefs d'œuvre non moins remarquables que l'Art moderne a produits ", notamment la basilique de Monreale et la Chapelle royale de Palerme, qui lui apparaissent comme « de véritables traditions de l'Art des Hellènes ". Cette fusion d'éléments anciens et modernes s'exprime également sur le plan technique : à l'emploi de la peinture à la cire, "précieux héritage de l'Antiquité ", de la peinture sur verre, "admirable invention de nos Ancêtres ", doit venir s'ajouter la peinture sur lave émaillée « qui réunit, à toutes les qualités de la mosaïque, un grand nombre d'avantages du plus haut intérêt. » Or cette dernière est le fruit d'une découverte très récente, puisque le procédé a été élaboré en

59 La lettre, publiée dans la revue L'Artiste en janvier 1842, car elle apparaît comme un véritable manifeste artistique, est reproduite dans Hittorff, p. 341-346. 
1827. Hittorff n'hésite donc pas à "moderniser les techniques picturales des Grecs ", comme il l'affirme lui-même ${ }^{60}$. Il a sans doute tiré la leçon de son examen des marbres d'Elgin, qui ont mis en évidence l'évanescence de la peinture et la fragilité des pigments : il s'agit donc de dépasser les Anciens, en produisant une polychromie stable et durable. Les plaques émaillées polychromes sont en effet destinées à la parure extérieure de l'édifice, celle qui est soumise aux intempéries. Hittorff prévoit de les placer à l'entrée, sous le porche, de chaque côté de la porte (fig. 1). Il réussit à faire accepter le projet et en 1860 sept plaques, représentant des scènes tirées de la Bible, sont posées sur les murs de la façade. Cependant le visiteur actuel risque aujourd'hui d'être déçu, en trouvant un porche qui fait grise mine (fig. 5), car elles furent vite retirées, sur ordre du baron Haussmann, alors préfet de la Seine. Les paroissiens étaient scandalisés par la nudité d'Adam et Ève tout autant que par l'intrusion violente et sensuelle de la couleur à l'entrée de l'édifice religieux 61 .

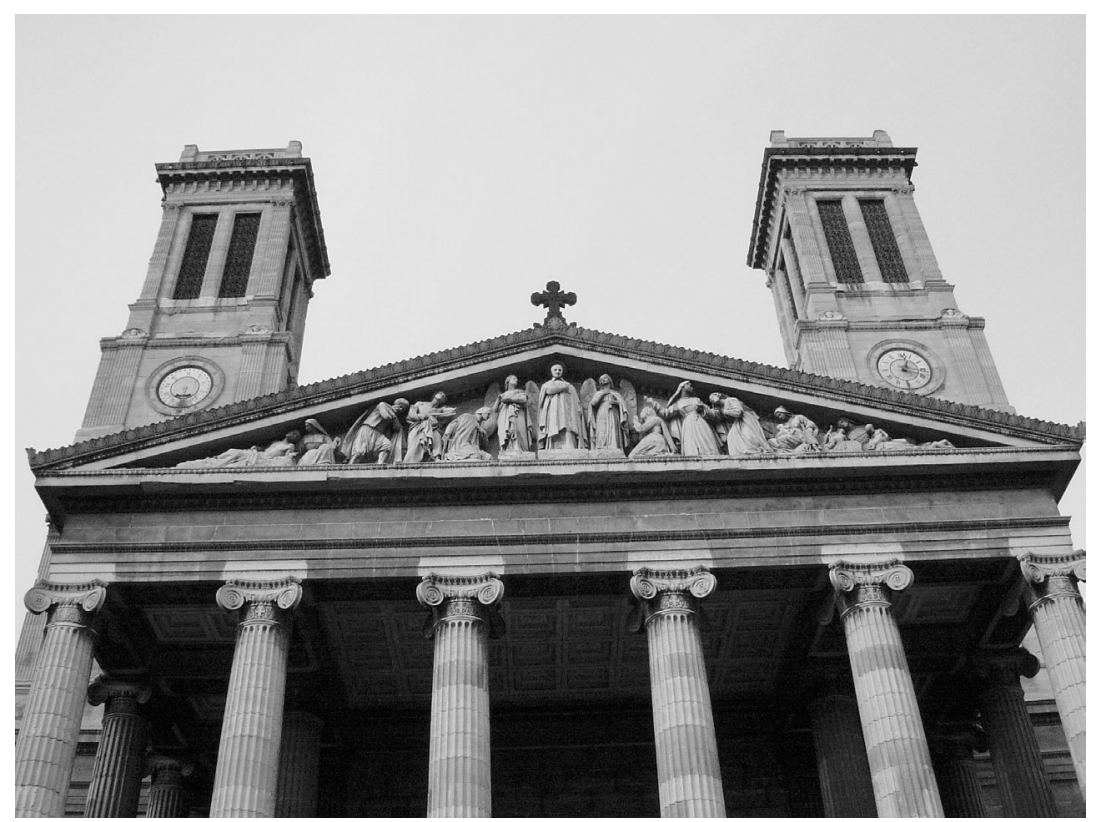

Figure 4. Façade actuelle de l'église St-Vincent de Paul, à Paris (CA. Grand-Clément).

60 Hittorff, p. 121.

61 Cf. Cl. de Vaulchier, "Hittorff et les laves émaillées 1832-1838", in Hittorff, p. 299. Pour un dessin du projet initial, cf. Hittorff, p. 153. 


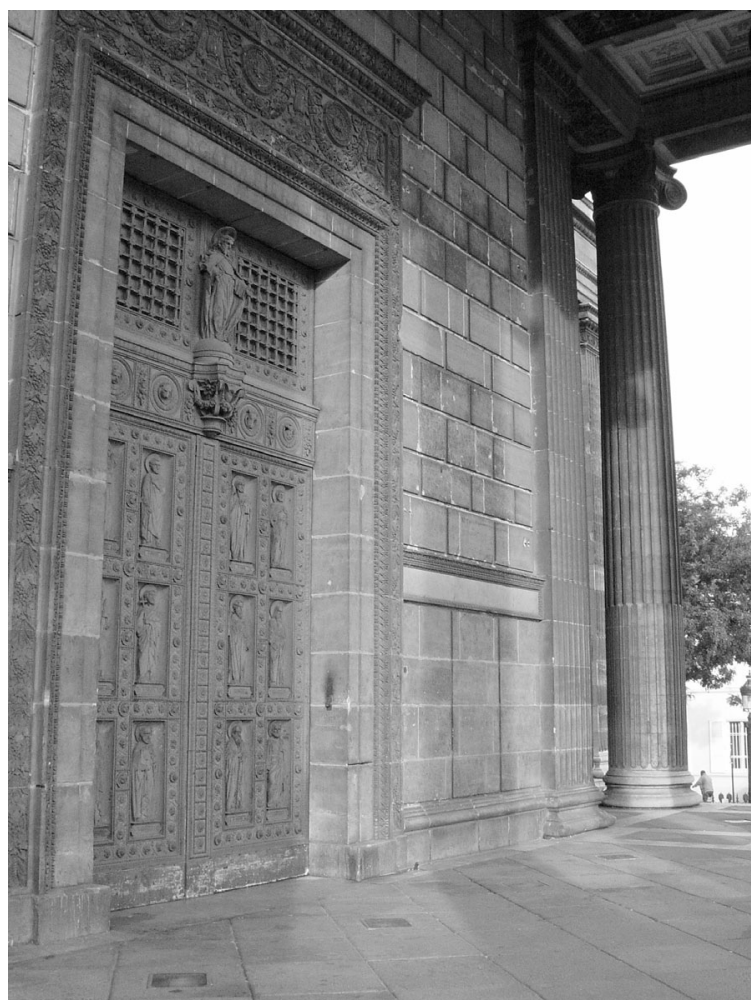

Figure 5. Aspect actuel du mur situé sous le porche de l'église St-Vincent de Paul et qui servait à l'origine de support à des plaques émaillées polychromes (CA. Grand-Clément).
$\mathrm{Si}$, pour faire accepter ses projets polychromes, Hittorff décide parfois d'invoquer le modèle antique, il le fait toujours avec pondération et ne se réclame pas uniquement de la tradition hellène. Il pressent que les principes de la polychromie architecturale n'ont cessé d'être appliqués au cours de l'histoire : l'Antiquité n'est plus alors l'unique modèle de référence, mais se trouve réinsérée dans une chaîne universelle, dont elle forme l'un des maillons relié d'un côté aux pratiques égyptiennes 62 et de l'autre à l'art médiéval. L'idée est développée à la fin du siècle par l'architecte Paul Sédille, qui reproche à l'art néo-classique son aridité monochrome ${ }^{63}$. Convaincu que l'enseignement de l'histoire et de l'archéologie doit peser sur le goût de l'époque, servir à éduquer les esprits et à faire accepter la polychromie monumentale, il affirme, lors d'une conférence prononcée en 1886 devant les

62 La riche polychromie des édifices égyptiens est connue depuis l'expédition scientifique qui a accompagné les troupes napoléoniennes. En 1822, F.-Chr. Gau, originaire comme Hittorff de Cologne, expose au salon de Paris une série d'études vivement colorées de façades et de tombes peintes égyptiennes. Il publie la même année d'excellentes planches chromolithographiées dans Les Antiquités de Nubie. Ces dessins d'édifices polychromes ont sans doute excité l'imagination de Hittorff. Celui-ci s'intéresse d'ailleurs à l'Égypte : chargé de l'aménagement de la place de la Concorde, il propose, pour accueillir l'obélisque offert à la France en 1831, de lui adjoindre un haut socle doré et une étoile dorée sur le pyramidion. Il étaye alors sa suggestion par un petit argumentaire historique : Précis sur les pyramidions en bronze doré employés par les anciens Égyptiens comme couronnements de quelques-uns de leurs obélisques, à l'appui de la proposition de restituer de la même manière le pyramidion de l'obélisque de Louqsor, Paris, Renouard, 1836. Son projet est finalement repoussé.

63 Cf. B. GiRveAU, "Sources et principes de la polychromie monumentale selon Paul Sédille (1836-1900)", Histoire de l'Art 39 (1997), p. 57-68. 
membres du RIBA à Londres, que la polychromie était un principe universel de l'architecture, éclipsé en France depuis le XVII e siècle ${ }^{64}$.

En définitive, Hittorff promeut-il la polychromie architecturale parce qu'il faut imiter en tous points les Anciens ? La réponse est négative. C'est ce que révèle un passage de son ouvrage de 1851, dans lequel il analyse avec beaucoup de lucidité le rapport complexe qui existe entre ses études sur l'Antiquité, son œuvre de savant, et ses réalisations architecturales :

Le principal but de mes études, qu'elles eussent pour objet les monuments antiques, a toujours été de chercher les éléments propres à me servir dans la carrière pratique de l'architecture. J'ai donc tâché, partout où la raison m'en démontrait la convenance, d'en traduire dans la distribution, dans les moyens de construction et de décoration des édifices que j'avais à élever, ce que les études me montraient d'un avantageux emploi ; mais ma sincère admiration pour les œuvres d'art antique ne m’a pas empêché de reconnaître dans beaucoup de productions des anciens ou une infériorité individuelle ou une influence étrangère à l'art et nuisible à sa perfection. Aussi n'ai-je jamais fait l'usage d'un élément antique parce que je l'avais vu employé par les artistes grecs et romains, mais parce que je l'avais jugé bien appliqué par eux et que son nouvel emploi devait, indépendamment de son origine, amener un résultat satisfaisant. Ce n'est point par suite de l'apparition de l'application des couleurs à la décoration extérieure des monuments de la Grèce et à cause de la nouveauté apparente de ce système que jai été porté à m’en servir au cirque et à son porche, mais par la raison que la peinture est à Paris plus qu'à Athènes un moyen de préservation des matériaux et que si des couleurs aident sous le ciel de Grèce, de la Sicile et de l'Italie à faire distinguer davantage et les sculptures et les parties les plus importantes des formes architectoniques, leur concours est sous ce rapport bien plus efficace et plus nécessaire sous un ciel privé de soleil 65 .

Hittorff souligne clairement que la référence antique ne fait pas office d'argument d'autorité intangible. Il est souhaitable que les usages des Anciens alimentent l'inspiration de l'artiste, mais ce dernier doit savoir y puiser de manière sélective, raisonnée et justifiée. L'archéologue explique l'origine de la polychromie antique par un double rôle, fonctionnel - préserver des édifices qui étaient à l'origine en bois - et esthétique - créer une harmonie avec un environnement naturel richement coloré ; l'architecte en tire des leçons et estime que, sur ces deux points, les conditions offertes par la ville de Paris, au XIX ${ }^{\mathrm{e}}$ siècle, rendent encore plus impérieuse la bigarrure monumentale.

Il est ardu, sinon impossible, de discerner ce qui, dans le parcours de Hittorff, relève de son expérience d'archéologue helléniste et ce qui appartient à son œuvre d'ar-

64 GIRVEAU, "Sources et principes de la polychromie monumentale...", p. 57.

65 HitTorfF, Restitution du temple d'Empédocle à Sélinonte, p. 814 ; c'est nous qui soulignons. 
chitecte. L'interpénétration constante entre les deux versants de son activité, en une sorte de fécondation croisée, dote son parcours d'une configuration particulière et annihile tout effort de catégorisation. Si, toute sa vie, il s'est voulu à l'école de la Grèce, depuis la formation qu'il a reçue, jusqu'aux projets architecturaux qu'il a dirigés, il a tracé la voie d'une réappropriation originale de l'héritage des Anciens, qui marque la fin de la référence exclusive et dogmatique à l'Antiquité classique. Son influence a profondément marqué la génération suivante d'architectes, tels Henri Labrouste et Charles Garnier. Hittorff a ainsi fait office de passeur, de médiateur entre le néo-classicisme et l'éclectisme ${ }^{66}: c^{\prime}$ 'est l'idée qu'a voulu exprimer Donald David Schneider, lorsqu'il le qualifie de " néo-classique éclectique 67 ».

Reprenons à présent la question de départ : comment Hittorff a-t-il réussi à conjuguer à la fois un approfondissement des connaissances sur l'architecture grecque et une prise de distance par rapport au modèle antique ? La réponse tient en fait au contexte : son activité intervient à un moment charnière, lorsque débute le divorce entre le monde des artistes et celui des archéologues. À partir de la deuxième moitié du XIXe siècle, chaque univers prend son autonomie et désormais les hellénistes n'ont plus besoin des artistes pour mener à bien leurs recherches : ils ont élaboré des outils d'investigation et des méthodes de travail qui leur sont propres. Hittorff représente donc l'un des derniers prototypes de l'architecte-archéologue, qui fut un temps la clef de voûte des progrès de la science archéologique européenne. C'est ce qui en fait une figure emblématique, comme le souligne avec force Beulé, dans le vibrant éloge funèbre qu'il prononce en sa mémoire, le 12 décembre 1868, devant l'Académie des Beaux-Arts :

Il était réservé au XIXe siècle d'apporter à l'étude de l'antiquité une pénétration et un respect qui font tout revivre. Les ruines de la Grèce et de l'Italie sont une école pour des générations entières ; les élèves les plus habiles vont recueillir, dans les pays déserts ou gardés par la fièvre, les moindres détails des monuments grecs et romains ; ils se nourrissent de ces œuvres à la fois si belles et si simples, ils les découvrent, ils les restaurent, ils les mesurent, ils en retournent le plus petit débris, comme un commentateur retourne les phrases d'un manuscrit précieux. En un mot, à force d'exactitude, de délicatesse, d'éclectisme, nos architectes ne se contentent plus d'être des savants; ils sont devenus des archéologues.

Celui que nous honorons aujourd'hui, Messieurs, a été un des promoteurs de ce triomphe de l'archéologie. Hittorff était un érudit autant qu'un artiste. L'Académie des Inscriptions et Belles Lettres aurait eu, autant que l'Académie des Beaux-Arts, le droit de l'appeler en son sein. S'il a aimé son art, il a cultivé la science avec ivresse et vécu au milieu de l'antiquité comme dans un sanctuaire. Il compte parmi les figures complexes et inté-

66 Cl. MignOT définit le courant éclectique comme un « renouvellement du classicisme par une utilisation plus libre de l'ensemble de la tradition gréco-romaine " (L'architecture au XIXe siècle, Fribourg, Éditions du Moniteur, 1983, p. 84). 
ressantes de notre époque, par la variété même de ses aptitudes et par la sincérité d'une passion qui a rempli toute sa vie. [...] C'est pourquoi, Messieurs, Hittorff marque dans l'art français du XIXe siècle; il y laisse son empreinte et comme artiste et surtout comme savant. [...] Le nom de Hittorff restera attaché au nom et à l'idée de la Polychromie ${ }^{68}$.

Vœu pieu que celui de Beulé, si l'on s'en tient à l'éclipse que connaît aujourd'hui la renommée de Hittorff... Gageons cependant que le renouveau des recherches actuelles sur la polychromie de l'art antique aura aussi le mérite de remettre à l'honneur ce grand savant.

\begin{tabular}{l} 
Adeline GRAND-CLÉMENT \\
\hline Maître de conférences en Histoire grecque \\
à l'Université de Toulouse-Le Mirail \\
24 rue des Braves \\
31300 Toulouse \\
adelinegc@yahoo.fr
\end{tabular}

68 Le texte figure dans Hittorff, p. 349-354. 